\title{
PROBABILISTIC LOAD FLOW FOR MV RADIAL DISTRIBUTION NETWORKS SUPPLYING STATISTICAL LOADS
}

\author{
B.J. Kundy \\ Department of Electrical Power Engineering, University of Dar es Salaam \\ B. O. Box 35131, Dar es Salaam, Tanzania.
}

\begin{abstract}
$A$ ccurate load models are required for the computation of load flows on Medium Voltage (MV) distribution networks. Modern microprocessors in recent times have enabled researchers to sample and log domestic loads. The findings show that domestic loads are stochastic in nature and are best described by a beta probability distribution [Herman et al, 1999]. The statistical evaluation of the consumer voltages requires a description of load currents at the time of the system maximum demand. In this paper, an analytical tool for computing voltage regulation on $M V$ distribution networks feeding statistical loads is presented. To deal with betadistributed currents on $M V$ distribution networks, new scaling factors are evaluated at each node. These new scaling factors are evaluated from the distribution transformer turns ratio and the deterministic component of the statistically distributed load currents treated as constant real power loads. The comparison between analytical and Monte Carlo simulation results compare very well giving a maximum percent difference voltage drop of $-0.0153 \%$ for the risk level of $10 \%$ and $20 \%$ from the test data.
\end{abstract}

Keywords: Probabilistic Load flow; Radial distribution networks; Statistical modeling

\section{INTRODUCTION}

The accuracy of the computations for power flow in distribution systems depends on how the loads are modelled. It needs to be said that the result from load flow studies will be regarded as useful if the load model and data used in the analysis are reasonably accurate. Significant research has been done and published in this area [IEEE, 1995]. The load models are categorised on the basis of their applications. Despite this wide coverage of load models, their application on MV distribution network systems feeding stochastic loads is inadequate. It is imperative that to obtain an accurate load description, application of end-use models are necessary. But, this requires a considerable amount of load-research data. In [Herman et al, 1993], the authors put emphasis on performing the analysis of the behaviour of domestic electrical loads on the basis of valid data measurement. In achieving this goal, means of recording load data coincidentally must be realised. In order to obtain reliable load data, they applied a modern microprocessor technology, which comprises of a multi-channel microprocessor based loggers equipped with an accurate on-board time clock. This enables the monitoring of the multiple consumer load currents coincidentally at regular intervals. Analysis to date indicates that the most appropriate statistical model for describing grouped domestic electrical loads is the beta probability density function (p.d.f.) [Herman et al, 1993].

In [Herman et al, 1998], the authors describe a probabilistic load-flow currently known as the Herman Beta method. The Herman Beta method of calculating voltage drops in LV feeders was developed for three-phase, four-wire and biphase topologies using the Beta pdf description for the load currents. The method uses the principle of manipulating random variables of current into voltage drop random variables. The statistical parameters of the consumer voltages are then evaluated from their first and second moments. The first and the second moments of the consumer voltages are expressed in terms of 
the first and the second moments of the branch voltage drops. The percentile value of the consumer voltage variable is obtained by assigning a risk level or conversely, a confidence level. The approach adopted in [Herman et al, 1998], proves to

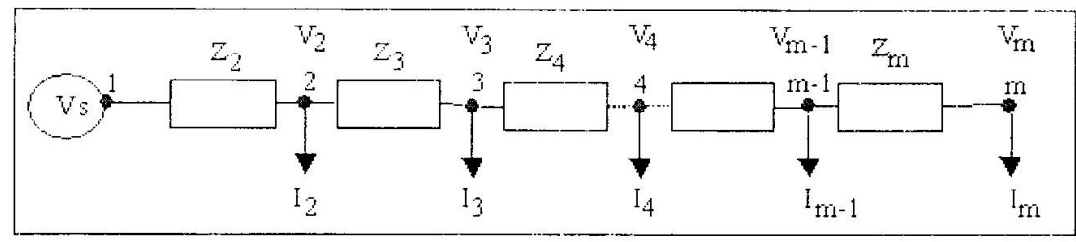

Figure 1: One line diagram of a short model distribution line. work well in developing the required general expressions. This is due to the fact that, the analysis is dealing with the three-phase fourwire system on the LV distribution networks where line inductive reactance and the consumer voltage phase angles are neglected. On MV distribution systems, such assumptions are not valid and therefore the analysis should take into consideration the line inductive reactance and the consumer voltage phase-angles. It should also be borne in mind that, expression adopted for the Taylor expansion would give pessimistic results in evaluating system voltage drop greater than $15 \%$. The analysis assumes three-phase balanced system and therefore per phase representation is considered.

\section{PROBABILISTIC LOAD FLOW ANALYSIS}

The probabilistic load flow analysis in this paper is based on the determination of the first and the second moments of the real and imaginary parts of the branch voltage drops due to statistical load currents as described in [Herman, 1993]. The first moments are obtained by considering the expected values of the individual load current variants that result from the total real and total imaginary components of the branch voltage drops at any node of the network. The second moments are obtained by considering the expected values of the individual load current variants that result from the square of the total real and total imaginary components of the branch voltage drops at any node of the network. Therefore, the development of the branch voltage drop equations is the prerequisite in order to apply probabilistic approach on the evaluation of the voltage profile along the MV radial distribution network without branches as depicted in Figure 1. In order to be able to derive the required equations, the load current phasors $I_{2}, I_{3}, I_{4}, I_{5} \ldots \ldots \ldots I_{m}$ in Figure 1 are assumed to have phase angles $\alpha_{2}, \alpha_{3}, \alpha_{4}, \alpha_{5} \ldots \ldots \ldots \ldots \ldots \alpha_{m}$ respectively. The node voltages at respectively nodes are designated $V_{k}$ for $k$ being the node number while the branch impedance between nodes $k$ and $k-1$ are designated as $Z_{k}$.

The consumer voltages are determined by taking the difference between the magnitude of the supply voltage $V_{S}$ and the total of the branch voltage drops from the supply node to the node under consideration. Therefore, the total real and total imaginary component of the branch voltage drops from the supply node with respect to the other system nodes should be determined. According to Figure 1, the total real and total imaginary components of the branch voltage drops at node $i$, can be expressed as:

$$
\begin{aligned}
& \nabla V_{\text {ireal } \ldots t}=\sum_{y=2}^{i} \sum_{k=y}^{m} I_{k}\left(R_{y} \cos \alpha_{k}-X_{y} \sin \alpha_{k}\right)(1) \\
& \nabla V_{\text {iimag }_{-t}}=\sum_{y=2}^{i} \sum_{k=y}^{m} I_{k}\left(R_{y} \sin \alpha_{k}+X_{y} \cos \alpha_{k}\right)(2)
\end{aligned}
$$

where

$R_{k} \quad$ is the resistance between nodes $k$ and $k-1$

$X_{k} \quad$ is the inductive reactance between nodes $k$ and $k-1$

$I_{k} \quad$ is the load current at node $k$

$\alpha_{k} \quad$ is the load current phase angle at node $k$

$\Delta V_{\text {ireul } . .,} \quad$ is the total real component of the branch voltage drop at node $i$

$\Delta V_{\text {immul }}, \quad$ is the total imaginary component of the voltage drop at node $i$

The square of expressions (1) and (2) can be expressed as: 


$$
\begin{aligned}
& \nabla V_{\text {tresil }}^{2} t=\sum_{y=2}^{i} \sum_{z}^{m} I_{z}^{2}\left(R_{y}^{2} \cos ^{2} \cos \alpha_{z}\right. \\
& \left.+X_{y}^{2} \sin ^{2} \alpha_{z}-2 R_{y} X_{y} \cos \alpha_{z} \sin \alpha_{z}\right) \\
& +2 \sum_{y=2}^{i-1} \sum_{k=1}^{m-1} \sum_{n=k+1}^{m} I_{h} I_{n}\left\{R_{y}^{2} \cos \alpha_{k} \cos \alpha_{n}\right. \\
& +X_{y}^{2} \sin \alpha_{k} \sin \alpha_{n}-R_{y} X_{y} \sin \left(\alpha_{k}+\alpha_{n}\right) ? \\
& +\sum_{i=2}^{i-1} \sum_{r=t+1}^{i} \sum_{k=1}^{m} \sum_{n=r}^{m} I_{k} I_{n}\left\{R_{t} R_{r} \cos \alpha_{k} \cos \alpha_{n}\right. \\
& +X_{1} X_{r} \sin \alpha_{k} \sin \alpha_{n} \\
& -R_{t} X_{r} \cos \alpha_{k} \sin \alpha_{n}-X_{t} R_{r} \sin \alpha_{k} \cos \alpha_{n} \\
& \nabla V_{\text {iimang } t}^{2}=\sum_{y=2}^{i} \sum_{z}^{m} I_{z}^{2}\left(R_{y}^{2} \sin ^{2} \alpha_{z}\right. \\
& \left.+X_{y}^{2} \cos ^{2} \alpha_{y}+2 R_{y} X_{y} \cos \alpha_{y} \sin \alpha_{y}\right) \\
& +2 \sum_{y=2}^{i-1} \sum_{k=1}^{m \cdot 1} \sum_{n=k+1}^{m} I_{k} I_{n}\left\{R_{y}^{2} \sin \alpha_{k} \sin \alpha_{n}\right. \\
& +X_{y}^{2} \cos \alpha_{k} \cos \alpha_{n}+R_{y} X_{y} \sin \left(\alpha_{k}+\alpha_{n}\right) ! \\
& +\sum_{t=2}^{i-1} \sum_{r=t+1}^{i} \sum_{k=1}^{m} \sum_{n=r}^{m} I_{k} I_{n}\left\{R_{t} R_{r} \sin \alpha_{k} \sin \alpha_{n}\right. \\
& +X_{i} X_{r} \cos \alpha_{k} \cos \alpha_{n}+R_{t} X_{r} \cdot \cos \alpha_{n} \sin \alpha_{k} \\
& +X_{t} R_{r} \cdot \sin \alpha_{n} \cos \alpha_{k}
\end{aligned}
$$

The consumer voltage $V_{s(m)}^{r}$, at node $i$, can be expressed as:

$$
\begin{aligned}
& V_{s c m, i}=\left\{\left(V_{S}^{r}-\Delta V_{\text {ireal } t}\right)^{2}+\left(\Delta V_{\text {immag }}\right)^{2}\right\} \\
& =V_{S}\left\{1 \cdot 2 \frac{\Delta V_{\text {ireal }}+}{V_{S}}+\frac{\Delta V^{2}{ }_{\text {ireti }}+}{V_{S}{ }^{2}}+\frac{\Delta V^{2}{ }_{\text {immas }}}{V_{S}{ }^{2}}-\right\}^{\frac{1}{2}}
\end{aligned}
$$

Using Taylor's expansion, equation (5) can be expressed in such a way that the first statistical moment of the consumer voltage $V_{\text {scon } ;}$ may be determined [Herman et al, 1994]. The Taylor series is described as:

$$
\begin{aligned}
& (1+X)^{\frac{1}{2}}=1+\frac{1}{2} X-\frac{1}{8} X^{2}+\frac{1}{16} X^{3} \ldots \ldots . . \\
& -1<X<1
\end{aligned}
$$

If equation (5) is normalised, the parameter $X$ in equation (G) can be given as:

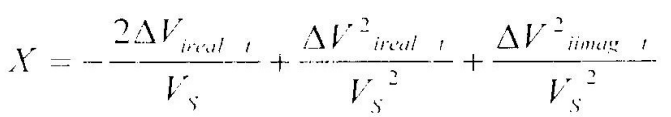

The terms $1+\frac{1}{2} X-\frac{1}{8} X^{2}+\frac{1}{16} X^{3}$ will be considered in the Taylor's expansion. The final result of the consumer voltage $V_{s i o n}$, at node $i$ after discarding powers greater than 2 , is approximated as:

$$
\begin{aligned}
& V_{s(\mathrm{~m}) \mathrm{i}} \approx
\end{aligned}
$$

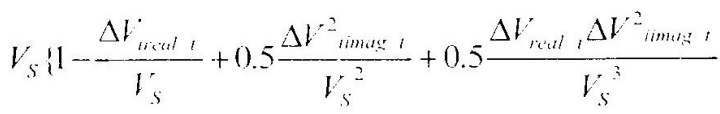

$$
\begin{aligned}
& +0.5 \frac{\Delta V^{2}{ }^{2} r_{w i}, \Delta V^{2}}{V_{S}{ }^{4}}
\end{aligned}
$$

In order to improve the accuracy of expression (8), the coefficients for the last three terms were evaluated with the aid of a search engine that was imbedded in the probabilistic load flow program. The final coefficients can be expressed as:

$$
\begin{aligned}
& V_{\text {seron_i }}=
\end{aligned}
$$

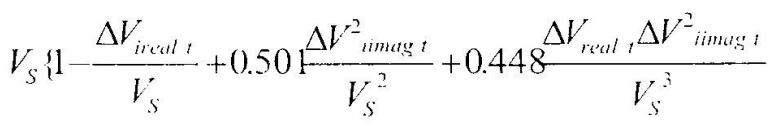

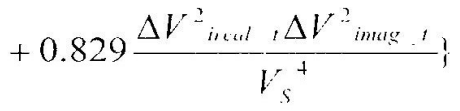

The square of the consumer voltage $V^{2}{ }_{\text {seon }} i$ is obtained by squaring equation (5).

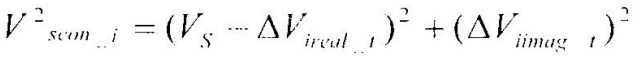

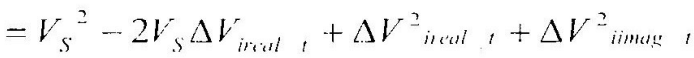

Due to the stochastic nature of the load currents, the consumer voltage distribution fit the beta model. Its statistical parameters can be derived from the first and the second moments of distribution [Herman, 1993].

\section{STATISTICAL ANALYSIS BASED ON THE BETA MODEL}

In statistics, if $Y_{1}, Y_{2}, Y_{3} \ldots \ldots . Y_{k}$ are independent variables and are identically distributed according to a beta probability distribution 
function having parameters $\alpha$ and $\beta$, it follows that the following expected values can be given as [Herman, 1993]:

$$
\begin{aligned}
& E\left[Y_{i}\right]=\frac{\alpha}{\alpha+\beta} \\
& E\left[Y_{i}, Y_{j}\right]=\frac{\alpha^{2}}{(\alpha+\beta)^{2}} \\
& E\left[Y_{i}^{2}\right]=\frac{\alpha(\alpha+1)}{(\alpha+\beta)(\alpha+\beta+1)}
\end{aligned}
$$

The parameters $\alpha$ and $\beta$ are evaluated from the mean and standard deviation of the measured data as follows:

$$
\begin{aligned}
& \alpha=\frac{\mu\left(c \mu-\mu^{2}-\sigma^{2}\right)}{c \sigma^{2}} \\
& \beta=\frac{(c-\mu)\left(c \mu-\mu^{2}-\sigma^{2}\right)}{c \sigma^{2}}
\end{aligned}
$$

where

$$
\begin{aligned}
& \mu \quad \text { is the mean value of the measured data } \\
& \sigma \quad \text { is the standard deviation of the measured } \\
& \text { data } \\
& c \quad \text { is the scaling factor conveniently taken } \\
& \text { as the rating of the circuit breaker }
\end{aligned}
$$

Suppose each load current in Figurel at node $i$ has $N_{i}$ electrical consumers. The current $I_{i}$ at node $i$ can be expressed as [Herman, 1993]:

$I_{i}=C B_{i}\left(Y_{1}+Y_{2}+Y_{3}+\ldots+Y_{V_{2}}\right)$

If all $Y^{\prime S}$ are independent and are identically distributed with parameters $\alpha_{i}$ and $\beta_{i}$, it follows that according to equation (11) and (16), the expected value of the current $I_{i}, E\left[I_{i}\right]$ will be:

$$
E\left[I_{i}\right]=C B_{i} N_{i}\left(\frac{\alpha_{i}}{\alpha_{i}+\beta_{i}}\right)
$$

The expected value of the square of the current $I_{i}, E\left[I_{i}^{2}\right]$ can be expressed as:

$$
\begin{aligned}
& E\left[I_{i}^{2}\right]=C B_{i}^{2}\left\{N_{i} \frac{\alpha_{i}\left(\alpha_{i}+1\right)}{\left(\alpha_{i}+\beta_{i}\right)\left(\alpha_{i}+\beta_{i}+1\right)}\right. \\
& \left.+N_{i}\left(N_{i}-1\right) \frac{\alpha_{i}^{2}}{\left(\alpha_{i}^{2}+\beta_{i}^{2}\right)}\right\}
\end{aligned}
$$

For the product of two load currents $I_{k}$ and $I_{n}$ due to $N_{k}$ and $N_{n}$ consumers that are not identically distributed, their expected value $E\left[I_{k} I_{n}\right]$ can be expressed as:

$$
\begin{aligned}
& E\left[I_{k} I_{n}\right]=C B_{k} C B_{n} E\left[Y_{k}\right] E\left[Y_{n}\right] N_{k} N_{n} \\
& =C B_{k} C B_{n} N_{k} N_{n}\left(\frac{\alpha_{k}}{\alpha_{k}+\beta_{k}}\right)\left(\frac{\alpha_{n}}{\alpha_{n}+\beta_{n}}\right)
\end{aligned}
$$

where

$C B_{k}$ and $C B_{n}$ are the scaling factors at nodes $k$ and $n$ respectively

In practice the scaling factors can be conveniently be chosen as the circuit-breaker size of the consumers [Herman et al, 1997].

\section{EVALUATION OF NEW SCALING FACTORS}

In order to apply the same statistical parameter of the beta-distributed currents described in the low voltage level on MV distribution networks, new scaling factors should be evaluated at each node. These new scaling factors are evaluated from the distribution transformer turns ratio and the deterministic component of the statistically distributed load currents treated as constant real power loads because domestic loads can be modelled as constant current at unity power factor [Herman, 1993]. The node phase angles assumed previously are calculated by deterministic load flow using constant real power loads as explained above and are considered to be constant in the probabilistic load flow analysis. The overall scaling factor, $S F$ at each load point taking into account the variable scaling factor $k_{\text {variuhte, }}$, the circuit breaker scaling factor $C B$ and the distribution transformer turns ratio $D_{t r}$ is evaluated as:

$S F_{h}=k_{\text {int } \mu_{h}} C B_{h} \cdot D_{\| \prime}$

where 
$k_{\text {variatle }}=\frac{I_{k-m m \cdot l v}}{I_{k-l, k}}$

$D_{t r}=400 / 11000$

$I_{k-l v}=C B_{k} N_{k} \frac{\alpha_{k}}{\alpha_{k}+\beta_{k}}$ is the deterministic value of beta-distributed currents at node $k$ on low voltage side

$I_{k-m,}$ is the current on MV side evaluated from the deterministic load flow

$I_{k-m v, t v}$ is the converted value of $I_{k-m}$ to the low voltage side of the distribution transformer $(11 \mathrm{kV} / 400 \mathrm{~V})$

By applying equations (11), (12) and (13), the expected values for expressions (1), (3) and (4) can be given as:

$$
\begin{aligned}
& E\left[\nabla V_{\text {ireal } t}\right]= \\
& \sum_{y=2}^{i} \sum_{k=y}^{m} S F_{k} N_{k} E\left[Y_{k}\right]\left(R_{y} \cos \alpha_{k}-X_{y} \sin \alpha_{k}\right) \\
& E\left[\nabla V_{\text {ireal }, t}^{2}\right]=\sum_{i=2}^{i} \sum_{==y}^{m} S F_{z}^{2} N_{z}\left\{E\left[Y_{z}^{2}\right]\right. \\
& +E\left[Y_{z}\right]^{2}\left(N_{z}-1\right)\left(R_{y}^{2} \cos ^{2} \alpha_{z}\right. \\
& \left.\left.+X_{y}^{2} \sin ^{2} \alpha_{z}-2 R_{y}, X_{y} \cos \alpha_{z} \sin \alpha_{z}\right)\right\} \\
& +\sum_{y=2}^{i} \sum_{k=y}^{m-1} \sum_{n=k+1}^{m} 2 S F_{k} S F_{n} E\left[Y_{k}\right] E\left[Y_{n}\right] N_{k} N_{n}\left\{R_{y}^{2} \cos \alpha_{k} \cos \alpha_{n}\right. \\
& \left.+X_{y}^{2} \sin \alpha_{k} \sin \alpha_{n}-R_{y} X_{y} \cdot \sin \left(\alpha_{k}+\alpha_{n}\right)\right\} \\
& +\sum_{i=2}^{i-1} \sum_{r=1+1}^{i} \sum_{n=r}^{m} S F_{n}^{2} N_{n}\left\{E\left[Y_{n}^{2}\right]+E\left[Y_{n}\right]^{2}\left(N_{n}-1\right)\right. \\
& \left(R_{i} R_{r} \cos ^{2} \cos \alpha_{n}+X_{t} X_{r} \sin ^{2} \alpha_{n}\right. \\
& \text {-- } \left.\left.R_{t} X_{r} \cos \alpha_{n} \sin \alpha_{n}-X_{t} R_{r} \sin \alpha_{n} \cos \alpha_{n}\right)\right\} \\
& +\sum_{i=2}^{i-1} \sum_{r=t}^{i} \sum_{k=t}^{m} \sum_{\substack{n=r \\
n \neq k}}^{m} S F_{k} S F_{n} E\left[Y_{k}\right] E\left[Y_{n}\right] N_{k} N_{n}\left(R_{i} R_{r}\right. \\
& \cos \alpha_{k} \cos \alpha_{n}+X_{t} X_{,} \sin \alpha_{k} \sin \alpha_{n} \\
& \left.-R_{t} X_{r} \cos \alpha_{k} \sin \alpha_{n}-X_{t} R_{r} \sin \alpha_{k} \cos \alpha_{n}\right)
\end{aligned}
$$

$$
\begin{aligned}
& E\left[\nabla V_{\text {imurg, }}^{2}\right]=\sum_{Y=2}^{1} \sum_{z=1}^{m} S F_{z}^{2} N_{z}\left\{E\left[Y_{z}^{2}\right]\right. \\
& +E\left[Y_{z}\right]^{2}\left(N_{z}-1\right)\left(R_{y}^{2} \sin ^{2} \alpha_{z}\right. \\
& \left.+X_{y}^{2} \cos ^{2} \alpha_{z}+2 R_{y} X_{y} \cos \alpha_{z} \sin \alpha_{z}\right) ! \\
& +\sum_{i=2}^{i} \sum_{k=1}^{m-1} \sum_{n=k+1}^{m} 2 S F_{k} S F_{n} E\left[Y_{k}\right] E\left[Y_{n}\right] N_{k} N_{n}\left\{R_{r}^{2} \sin \alpha_{k}\right. \\
& \left.\sin \alpha_{n}+X_{y}^{2} \cos \alpha_{k} \cos \alpha_{n}+R_{y} X_{y} \sin \left(\alpha_{k}+\alpha_{n}\right)\right\} \\
& +\sum_{i=2}^{i-1} \sum_{r=i+1 n=r}^{i} \sum_{n}^{m} S F_{n}^{2} N_{n}\left\{E\left[Y_{n}^{2}\right]+E\left[Y_{n}\right]^{2}\left(N_{n}-1\right)\right. \\
& \left(R_{t} R_{r} \sin ^{2} \alpha_{n}+X_{t} X_{r} \cos ^{2} \alpha_{n}\right. \\
& \left.\left.+R_{t} X_{r} \cos \alpha_{n} \sin \alpha_{n}+X_{r} R_{r} \sin \alpha_{n} \cos \alpha_{n}\right)\right\} \\
& +\sum_{i=2}^{i-1} \sum_{r=i+1}^{i} \sum_{k=1}^{m} \sum_{\substack{n=r \\
n \neq k}}^{m} S F_{k} S F_{n} E\left[Y_{k}\right] E\left[Y_{n}\right] N_{k} N_{n}\left(R_{t} R_{r}\right. \\
& \sin \alpha_{k} \sin \alpha_{n}+X_{t} X_{,} \cos \alpha_{k} \cos \alpha_{n} \\
& \left.+R_{t} X_{r} \cos \alpha_{n} \sin \alpha_{k}+X_{t} R_{r} \sin \alpha_{n} \cos \alpha_{h}\right)
\end{aligned}
$$

The general expression of the first and second moment of the consumer voltage distribution $V_{i c m}$ at node $i$, can be determined by finding the expected values of equations (9) and (10) respectively. When dealing with beta distribution function, the magnitude of the random variable should lie in the interval $(0,1)$ and having positive statistical parameters $\alpha_{1}$ " and $\beta_{v}{ }^{\ddot{ }}$ [Johnson et al, 1970]. Due to these conditions, the consumer voltage random variable $V_{s c o n} i$ at node $i$, should be scaled or normalised in order to satisfy the magnitude criteria and the same time rendering positive statistical parameters $\alpha_{v i}{ }^{\#}$ and $\beta_{1 i}{ }^{*}$. The scaling factor adopted in this paper is the MV nominal voltage or the operating voltage $V_{S}$. Therefore, the normalised consumer voltage $V^{*}{ }_{i c m}$ at node $i$ can be expressed as:

$$
V_{i<m n}^{=}=\frac{V_{i(u+n}}{V_{S}}
$$

Referring to equation (11), the expected value of the normalised consumer voltage $E\left[V_{i(t) i n}^{*}\right]$ can be expressed as: 


$$
E\left[V_{i m+m}^{\#}\right]=\frac{\alpha_{1 i}{ }^{*}}{\alpha_{1 i}{ }^{*}+\beta_{1 i}{ }^{*}}
$$

It follows that according to equation (13), the $2^{\text {mit }}$ moment of the scaled consumer voltage $E\left[V^{H 2}{ }_{i c m}\right]$ can be expressed as:

$$
E\left[V_{i c m u}^{\neq 2}\right]=\frac{\alpha_{1 i}{ }^{*}\left(\alpha_{1 i}{ }^{*}+\beta_{1 i}{ }^{\prime \prime}\right)}{\left(\alpha_{v i}{ }^{*}+\beta_{1 i}{ }^{*}\right)\left(\alpha_{1 i}{ }^{*}+\beta_{1 i}{ }^{*}+1\right)}
$$

Referring to [Herman et al, 1998], the statistical parameters $\alpha_{1 i}{ }^{*}$ and $\beta_{1 i}{ }^{*}$ of the scaled consumer voltage at node $i$, can be expressed as:

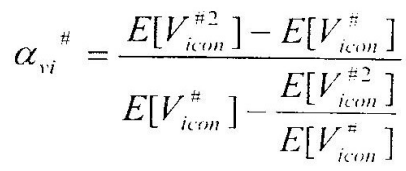

$$
\begin{aligned}
& \beta_{v i}{ }^{*}=\frac{\alpha_{v i}{ }^{\#}}{E\left[V_{i c m m}^{\#}\right]}-\alpha_{v i}{ }^{\prime \prime}
\end{aligned}
$$

According to equation (9), the expected value of the normalised consumer voltage $E\left[V_{i(i, n)}^{*}\right]$ at node $i$, can be given as:

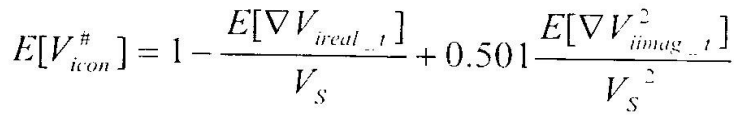

$$
\begin{aligned}
& +0.448 \frac{E\left[\nabla V_{\text {ireal_ }_{-}}\right] E\left[\nabla V^{2}{ }_{\text {iimmag, total }}\right]}{V_{S}{ }^{3}} \\
& +0.829 \frac{E\left[\nabla V^{2}{ }_{\text {ireal_trut }}\right] E\left[\nabla V_{\text {imurge }}^{2}\right]}{V_{S}{ }^{4}}
\end{aligned}
$$

Also, from equation (10), the second moment of the normalised consumer voltage $E\left[V_{i c u n}^{* 2}\right]$ at node $i$, can be given as:

$$
\begin{aligned}
& E\left[V_{k+m}^{\# 2}\right]=1-2 \frac{E\left[\nabla V_{\text {ireal }, t}\right]}{V_{S}}+\frac{E\left[\nabla V_{\text {ireal }}^{2},\right]}{V_{S}{ }^{2}} \\
& +\frac{E\left[\nabla V_{\text {iimug, }}^{2}\right]}{V_{S}^{2}}
\end{aligned}
$$

The percentile value of the consumer voltage $V_{i c u n}^{*}$ at node $\mathrm{i}$ can be obtained by applying a built in function BETAINV in MATLAB software package at a specified level of risk $R$ as:

$$
V_{i c o n}^{*}=\operatorname{BETAINV}\left(R, \alpha_{1 i}{ }^{*}, \beta_{1 i}{ }^{*}\right)
$$

Finally, the actual consumer voltage $V_{k m}$ at node $i$ at a specify level of risk, is calculated through rescaling the consumer voltage $V^{\text {if }}$ som $i$ of equation (24). Therefore, it can be expressed as:

$$
V_{i c m m}=V_{i c(c) !}^{*} * V_{S}
$$

\section{APPLICATION OF THE DEVELOPED GENERAL EXPRESSIONS}

In (Kundy, 2003), a development of a formulation for radial distribution network is presented. It shows that the structure of radial power distribution network allows each load point to be traced to the source. Having this characteristic, it is possible to develop a formulation that can relate each node with the source. The idea is to express the relationship in terms of the system currents, which means that, the individual load current is traced to the source. This is facilitated by the formulation of four main arrays as follows:

- path-array describing the path from any node to the supply node, this path contains all the branches connected between the node point in question and the supply node

- b-array describing the individual load currents through any branch of the network

- $x 2$-array containing the total number of paths (i.e. total number of connected branches) from any node of the network to the supply node

- wx-array containing the total number of load currents through any branch of the network 
Table 1: The line data and load data of Figure 2.

\begin{tabular}{|c|c|c|c|c|c|c|c|}
\hline branch & $R(\Omega)$ & $X(\Omega)$ & $\begin{array}{c}\text { node } \\
\text { no. }\end{array}$ & $\begin{array}{c}\text { no. of } \\
\text { consumers } \\
\text { per phase }\end{array}$ & $\alpha$ & $\beta$ & $\begin{array}{l}C B \text { for each } \\
\text { consumer }\end{array}$ \\
\hline $1-2$ & 15.6 & 7.414 & 1 & & & & \\
\hline $2-3$ & 15.6 & 7.414 & 2 & & & & \\
\hline $2-5$ & 15.6 & 7.414 & 3 & & & & \\
\hline $2-8$ & 15.6 & 7.414 & 4 & & & & \\
\hline $3-4$ & 15.6 & 7.414 & 5 & 40 & 0.68 & 8.95 & 63 \\
\hline $3-6$ & 15.6 & 7.414 & 6 & 40 & 0.68 & 8.95 & 63 \\
\hline $3-9$ & 15.6 & 7.414 & 7 & 40 & 0.68 & 8.95 & 63 \\
\hline $4-7$ & 15.6 & 7.414 & 8 & 40 & 0.68 & 8.95 & 63 \\
\hline $4-10$ & 15.6 & 7.414 & 9 & 40 & 0.68 & 8.95 & 63 \\
\hline \multicolumn{3}{|c|}{$3 \phi$ supply $=11 \mathrm{kV}$} & 10 & 40 & 0.68 & 8.95 & 63 \\
\hline
\end{tabular}

Table 2: The line and load data of Figure 2.

\begin{tabular}{|c|c|c|c|c|c|c|c|}
\hline branch & $R(\Omega)$ & $X(\Omega)$ & $\begin{array}{c}\text { node } \\
\text { no. }\end{array}$ & $\begin{array}{l}\text { no. of } \\
\text { consumers } \\
\text { per phase }\end{array}$ & $\alpha$ & $\beta$ & $\begin{array}{l}C B \text { for each } \\
\text { consumer }(A)\end{array}$ \\
\hline $1-2$ & 12.48 & 5.9312 & 1 & & & & \\
\hline $2-3$ & 12.48 & 5.9312 & 2 & & & & \\
\hline $2-5$ & 12.48 & 5.9312 & 3 & & & & \\
\hline $2-8$ & 12.48 & 5.9312 & 4 & & & & \\
\hline $3-4$ & 12.48 & 5.9312 & 5 & 40 & 1.2 & 10.4 & 63 \\
\hline $3-6$ & 12.48 & 5.9312 & 6 & 40 & 1.2 & 10.4 & 63 \\
\hline $3-9$ & 12.48 & 5.9312 & 7 & 40 & 1.2 & 10.4 & 63 \\
\hline $4-7$ & 12.48 & 5.9312 & 8 & 40 & 1.2 & 10.4 & 63 \\
\hline $4-10$ & 12.48 & 5.9312 & 9 & 40 & 1.2 & 10.4 & 63 \\
\hline \multicolumn{3}{|c|}{$3 \phi$ supply $=11 \mathrm{kV}$} & 10 & 40 & 1.2 & 10.4 & 63 \\
\hline
\end{tabular}

The entire distribution network can be described by an array ealled "D-array" in which the total number of columns represents the total number of the branches and their identification.

The developed general expressions $(21-32)$ to be used to perform the probabilistic load flow on MV radial distribution networks are derived from a radial distribution network without branches as depicted in Figure 1. In reality, MV distribution networks are all branched systems. The above mentioned formulation can be applied to make the developed expressions to be applicable on MV radial distribution network of any configuration. The procedure is facilitated by the developed arrays, which treats any distribution network as if it is not a branched system. This is possible by tracking the load currents flowing through the network branches between any node point and the supply node.

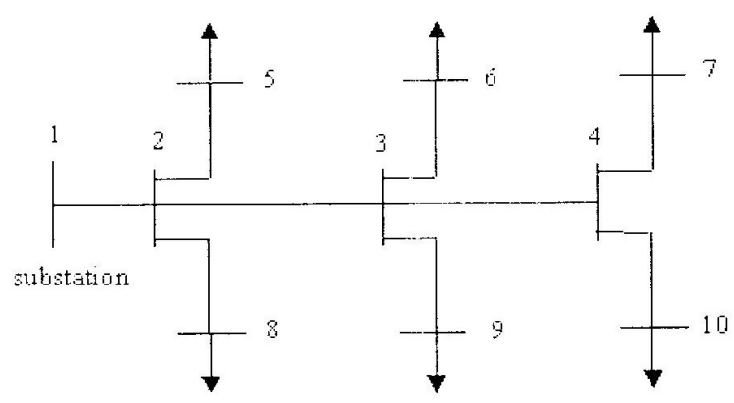

Figure 2:A single line diagram of an MV radial distribution testing network

\section{VERIFICATION OF THE ALGORITHM}

The consumer voltage percentile values are evaluated using the analytical procedure presented in previous sections based on the one line diagram depicted in Figure 2. 
The line and load data applied to test the developed algorithm is presented in Table 1 and Table 2.

Table 3: The comparison of consumer voltages for the data shown in Table 1.

\begin{tabular}{cccc}
\hline node & \multicolumn{3}{c}{ Risk $=10 \%$} \\
\cline { 2 - 4 } no: & Analytical & MC & \% \\
& (Volts) & simulation & difference \\
& & Volts & volt-drop \\
2 & 5470.1 & 5471.8 & -0.0005 \\
3 & 4859.0 & 4862.5 & -0.0011 \\
4 & 4540.2 & 4545.4 & -0.0018 \\
5 & 5338.8 & 5341.1 & -0.0007 \\
6 & 4711.0 & 4713.9 & -0.0010 \\
7 & 4379.9 & 4387.0 & -0.0025 \\
8 & 5338.8 & 5342.3 & -0.0010 \\
9 & 4711.0 & 4714.4 & -0.0011 \\
10 & 4379.9 & 4386.1 & -0.0022 \\
\hline
\end{tabular}

The probabilistic load flow was performed according to the computer flow chart shown in appendix-A(a). The analytical results obtained are compared with results from Monte Carlo simulation. A computer flow chart for the simulation procedure from beta distributed load currents is shown in appendix-A(b). The deterministic power load flow was performed using forward a backward sweep algorithm [Baran et al, 1997].

\section{EVALUATION CRITERION}

Monte Carlo simulation results are treated as benchmark values in calculating the \% difference volt-drop given as:

$\Delta V_{d r o p)} \%=\frac{\Delta V_{m c}-\Delta V_{u n}}{\Delta V_{m i c}} * 100$

where

$\Delta V_{m c}=\frac{V_{m p}-V_{m c}}{V_{o p}} * 100$ is the $\%$ voltage drop due to MC simulation

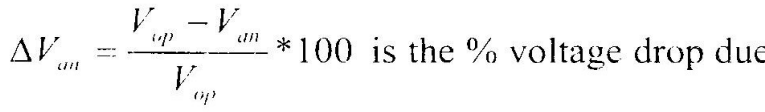
to analytical method

$V_{m} \quad$ is the consumer voltages due to $\mathrm{MC}$ simulation

$V_{i n}$ is the consumer voltages due to analytical method

$V_{u p} \quad$ is the operating phase voltage equal to 6350.9 Volts

The distribution network shown in Figure 2 was applied to evaluate the \% difference volt-drop at each node of the network. The line and load data used are shown in Table 1 and 2. This data gives a voltage drop of up to about $40 \%$ so that the accuracy of the proposed algorithm can be tested. The number of simulations employed for each case is 15000 in order to achieve a reasonable accuracy in evaluating the consumer voltages from Monte Carlo simulation. The results are shown in Table 3 and Table 5 for the risk level of $10 \%$ and Table 4 and Table 6 for the risk level of $20 \%$.

Table 4: The comparison of consumer voltages for the data shown in Table 1.

\begin{tabular}{cccc}
\hline node & \multicolumn{3}{c}{ Risk=20\% } \\
\cline { 2 - 4 } no: & $\begin{array}{c}\text { Analytical } \\
\text { (Volts) }\end{array}$ & $\begin{array}{c}\text { MC } \\
\text { simulation } \\
\text { Volts }\end{array}$ & $\begin{array}{c}\text { \% difference } \\
\text { volt-drop }\end{array}$ \\
& & 5499.9 & -0.0007 \\
2 & 5497.6 & 4908.5 & -0.0006 \\
3 & 4906.6 & 4604.1 & -0.0013 \\
4 & 4600.4 & 5373.2 & -0.0006 \\
5 & 5371.0 & 4768.2 & -0.0013 \\
6 & 4764.3 & 4451.7 & -0.0021 \\
7 & 4445.8 & 5373.8 & -0.0008 \\
8 & 5371.0 & 4768.3 & -0.0013 \\
9 & 4764.3 & 4452.1 & -0.0022 \\
\hline 10 & 4445.8 &
\end{tabular}


Table 5: The comparison of consumer voltages for the data shown in Table 2.

\begin{tabular}{cccc}
\hline node no: & \multicolumn{3}{c}{ Risk=10\% } \\
\cline { 2 - 4 } & $\begin{array}{c}\text { Analytical } \\
\text { (Volts) }\end{array}$ & $\begin{array}{c}\mathrm{MC} \\
\text { simulation } \\
\text { Volts }\end{array}$ & $\begin{array}{c}\% \\
\text { differen } \\
\text { ce volt- } \\
\text { drop }\end{array}$ \\
& & 5228.6 & -0.0008 \\
2 & 5226.1 & 4436.5 & -0.0026 \\
3 & 4429.1 & 4020.7 & -0.0086 \\
4 & 3998.8 & 5071.7 & -0.0008 \\
5 & 5069.2 & 4249.8 & -0.0042 \\
6 & 4238.4 & 3814.8 & -0.0153 \\
7 & 3777.7 & 5071.2 & -0.0006 \\
8 & 5069.2 & 4250.9 & -0.0046 \\
9 & 4238.4 & 3814.7 & -0.0153 \\
10 & 3777.7 & & \\
\hline
\end{tabular}

Table 6: The comparison of consumer voltages for the data shown in Table 2.

\begin{tabular}{cccc}
\hline node & \multicolumn{3}{c}{ Risk $=20 \%$} \\
\cline { 2 - 4 } & $\begin{array}{c}\text { Analytical } \\
\text { (Volts) }\end{array}$ & $\begin{array}{c}\text { MC } \\
\text { simulation } \\
\text { Volts }\end{array}$ & $\begin{array}{c}\% \\
\text { difference } \\
\text { volt-drop }\end{array}$ \\
2 & 5252.5 & 5254.7 & -0.0007 \\
3 & 4476.3 & 4481.7 & -0.0019 \\
4 & 4062.9 & 4079.6 & -0.0064 \\
5 & 5099.5 & 5102.5 & -0.0009 \\
6 & 4292.4 & 4301.8 & -0.0034 \\
7 & 3853.0 & 3880.1 & -0.0110 \\
8 & 5099.5 & 5102.2 & -0.0008 \\
9 & 4292.4 & 4301.4 & -0.0033 \\
10 & 3853.0 & 3879.5 & -0.0108 \\
\hline
\end{tabular}

\section{DISCUSSION OF THE RESULTS}

The data applied enables calculation of consumer voltage percentile values of about $60 \%$ of the operating voltage. These values were chosen so that the algorithm developed in this paper can be tested for its accuracy. The consumer voltage values evaluated at $10 \%$ risk (or $90 \%$ confidence level) can be interpreted as values that have 0.1 probability of being less than calculated values. Monte Carlo simulation and analytical results for the consumer voltage percentile values compare very well in the case of $10 \%$ and $20 \%$ risk level giving a maximum percentage difference voltage drop of -
$0.0153 \%$. These results show that the proposed algorithm can be applied to practical networks.

\section{CONCLUSION}

The algorithm developed in this paper can be applied to evaluate consumer percentile voltages on MV radial distribution networks using beta distributed load currents for a specified level of risk. The assumptions applied such as treating the nodal phase angles constant and the deterministic component of the statistically distributed load currents as constant real power loads had a little effect on the accuracy of the algorithm. Despite of the big range of consumer voltages considered, the algorithm demonstrated to be capable of producing acceptable results if beta-distributed load currents are applied to evaluate consumer voltages on MV radial distribution networks.

\section{NOMENCLATURE}

$E[Y]$ is the expected value of $Y$

p.d.f. is the beta probability density function

$S F_{k} \quad$ is the overall scaling factor

$V_{\text {stom } i} i$ is the first statistical moment of the consumer voltage $(V)$

$X \quad$ is the parameter used in the Taylor series

$\alpha \quad$ is the parameter of the beta probability distribution function

$\beta \quad$ is the parameter of the beta probability distribution function

$V_{i c o n}^{*}$ is the normalised consumer voltage at node $i(V)$

$V_{i c m}$ is the actual consumer voltage $V_{i c m}$ at node $i(V)$

$\alpha_{v i}{ }^{\prime \prime} \quad$ is the statistical parameters of the scaled consumer voltage at node $i$

$\beta_{v i}{ }^{\prime \prime} \quad$ is the statistical parameters of the scaled consumer voltage at node $i$ 

$\Delta V_{i r t a l}$, is the total real component of the branch voltage drop at node $i(V)$
$\Delta V_{\text {immug }}, \quad$ is the total imaginary component of the voltage drop at node $i(V)$
$\nabla V_{\text {ireal }}^{2}, \quad$ is the square of the total real component of the branch voltage drop at node $i\left(V^{2}\right)$
$\nabla V_{\text {iimmag. }}^{2}$ is the square of the total imaginary component of the branch voltage drop at node $i$ $\left(V^{2}\right)$

\section{REFERENCES}

1. B.J. Kundy, "Development of a Formulation for Radial Distribution Network, ComputerBased Analyses", ICEET 2003 Conference, University of Dar es Salaam, Tanzania, 2003, pp EP-11 - EP-17.

2. IEEE Task Force on Load Representation for Dynamic Performance, "Bibliography on Load Models for Power Flow and Dynamic Performance Sinulation", IEEE Transactions on Power Systems, Vol. 10, No. 1, February 1995, pp 523-538.

3. M.E. Baran, E.A. Staton, "Distribution Transformer Models for Branch Current Based Feeder Analysis", IEEE Transactions on Power Systems, Vol. 12, No. 2, May 1997, pp. 698-703.

4. N. I. Johnson and S. Kotz, "Continuous Univariate Distribution -- 2", Houghton Miffin, 1970, pg. 40.

5. R. Herman, "Voltage Regulation Analysis for the Design of Low Voltage Networks Feeding Stochastic Domestic Electrical Loads", Ph.D. thesis, University of Stellenbosch, November 1993.

6. R. Herman, J.J. Kritzinger, "The Statistical Description of Grouped Domestic Electrical Load Currents," Electric Power Research, 27, 1993, pp. 43-48.

7. R. Herman, S.W. Heunis, "General Probabilistic Voltage Drop Calculation Method for LV Distribution Networks Based on a Beta p.d.f. Load Model", Electric Power Systems Research 46 (1998) pp 45-49.

8. R. Herman, J.S. Maritz, "Voltage Regulation Algorithm for a Bi-phase Distribution System Feeding Residential Using a Beta p.d.f. Load Model", Electric Power Systems Research 43, 1997, pp. 77-80.

9. R. Herman, J.S. Maritz, J.H.R. Enslin, "The Analysis of Voltage Regulation in Residential Distribution Networks Using the Beta Distribution Model", Electric Power Systems Research 29 (1994) pp 213-216. 


\section{APPENDIX-A}

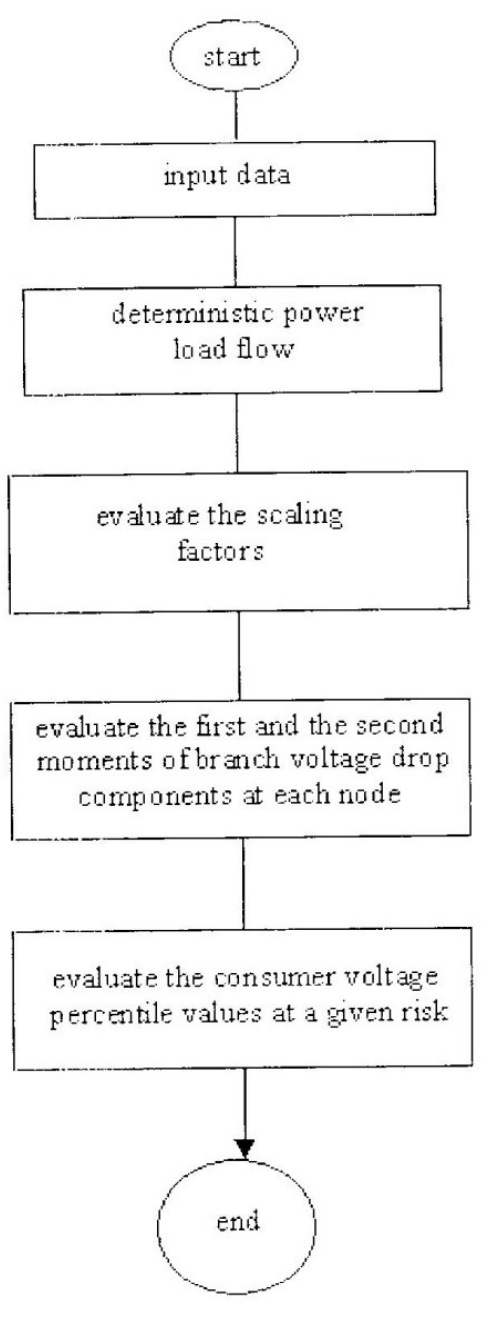

(a)

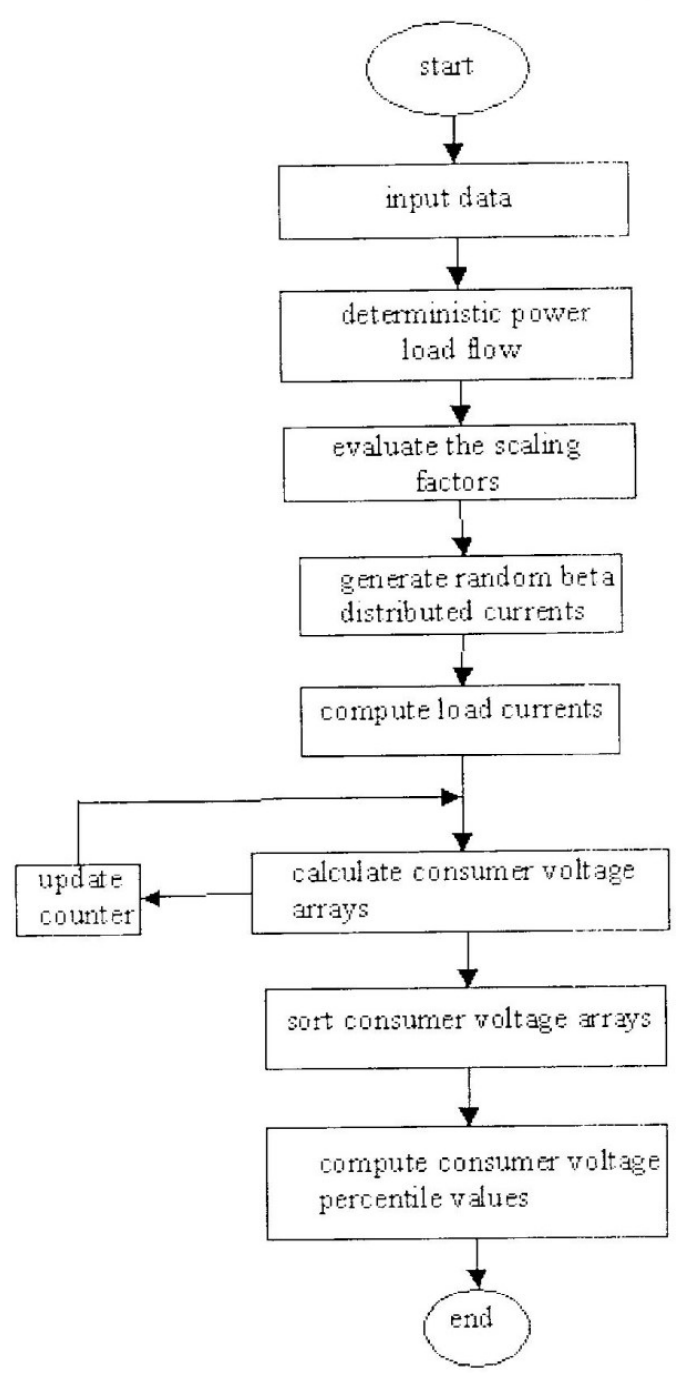

(b)

Figure 3: A computer flow chart for: (a) probabilistic load flow (b) MC simulations of the consumer voltages 